\title{
Modelo dinámico para el despliegue automático de una barrera de contención de vertidos marinos
}

\author{
Juan Jiménez \\ Universidad Complutense de Madrid, juan.jimenez@fis.ucm.es \\ Jose María Girón-Sierra \\ Universidad Complutense de Madrid, gironsi@fis.ucm.es \\ Dictino Chaos \\ Universidad Nacional de Educación a Distancia dchaos@dia.uned.es
}

\section{Resumen}

El presente trabajo presenta un modelo dinámico para el estudio del movimiento de una barrera de contención de vertidos marinos, cuando es remolcada por un par de barcos. Se describen los fundamentos seguidos en el modelado de la barrera, así como en el de los barcos remolcadores. A continuación, se presentan algunos escenarios básicos de remolque de las barreras, así como las implicaciones para el control del movimiento coordinado de los barcos que la remolcan. Por último, se presentan algunas conclusiones y posibles líneas de trabajo futuras.

Palabras clave: Vehículos Marinos, modelado de sistemas dinámicos, barreras de contención de vertidos, control cooperativo.

\section{Nomenclatura}

$\alpha_{b} \quad$ Velocidad angular $(\mathrm{rad} / \mathrm{s})$

$\mu_{a} \quad$ Coef. amort. lineal guiñada $(N \cdot m \cdot s)$

$\mu_{l}, \mu_{l 2} \quad$ Coef. amort. lineal $(N \cdot s / m)$ y cuadrático $\left(N \cdot(s / m)^{2}\right)$ avante

$\mu_{t}, \mu_{t 2} \quad$ Coef. amort. lineal $(N \cdot s / m)$ y cuadrático $\left(N \cdot(s / m)^{2}\right)$ deriva

$\theta_{b} \quad$ Rumbo (rad)

$\vec{n}_{i}, \vec{p}_{i} \quad$ Vectores unitarios normal y paralelo al elemento $i$

$\vec{T}_{i, i+1}$ Tensión entre elementos $i$ e $i+1$ de la barrera

$\vec{v}_{b} \quad$ Velocidad $(m / s)$

$a_{b x}, a_{b y}$ Componentes aceleración $\left(\mathrm{m} / \mathrm{s}^{2}\right)$

$F_{e x}, F_{e y}$ Componentes fuerza externa $(N)$

$F_{m x}, F_{m y}$ Componentes propulsión $(N)$

$I_{b} \quad$ Momento de inercia $\left(\mathrm{kg} \cdot \mathrm{m}^{2}\right)$

$l_{s} \quad$ Eslora $(m)$
$M \quad$ Momento aplicado por sistema de gobierno $(N \cdot m)$

$m_{b}, m_{b \|}, m_{b \perp}$ Masa y masas añadidas en avante y deriva, USV $(k g)$

$M_{e} \quad$ Momento externo aplicado $(N \cdot m)$

$m_{A i} \quad$ Masa y masa añadidas elemento $i(K g)$

$1 \quad$ Longitud de un elemento de barrera $(m)$

\section{Introducción}

El vertido al mar de sustancias contaminantes, como consecuencia de accidentes de buques de transporte o plataformas marinas de extracción, supone un serio problema que ha llevado a gobiernos y empresas a la búsqueda de medios, tanto para evitar que se produzcan como para minimizar su impacto una vez producidos [3].

Uno de los medios mecánicos más comúnmente usados, tanto para recoger un vertido como para confinarlo in situ y evitar su expansión, es el uso de barreras de contención [1], [7], [4]. Básicamente una barrera de contención consiste en una cadena de elementos compuestos por un flotador, un faldón impermeable y un balasto que mantiene el faldón sumergido, impidiendo así el paso de fluidos.

La fotografía de la figura 1 muestra una típica barrera de contención desplegada.

Las características de los materiales así como las dimensiones de la barrera vienen determinadas por la aplicación concreta a la que está destinada. En líneas muy generales se pueden distinguir barreras para aguas litorales y barreras para trabajos en alta mar. Las primeras se despliegan y se anclan para confinar un vertido o evitar que penetre en una zona de especial interés; mientras que las segundas son remolcadas para recoger un vertido o quemarlo in situ. La longitud de una barrera de contención puede variar entre 25 y 400 metros, y la profundidad del faldón entre 50 y 70 centímetros. 


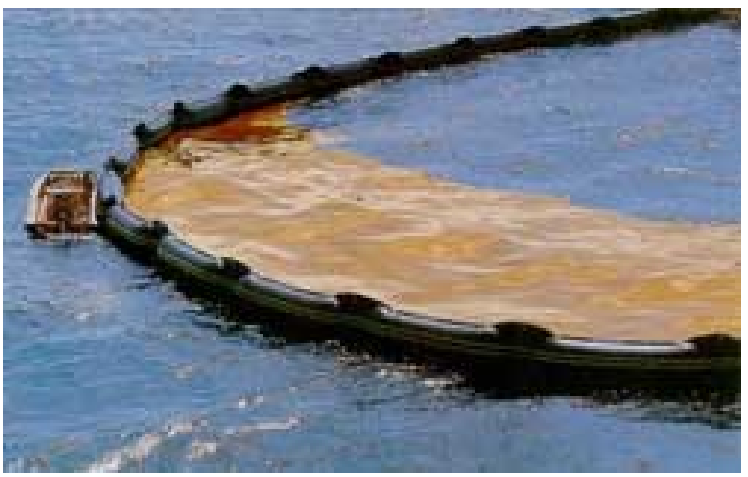

Figura 1: Imagen de una barrera de contención de vertidos desplegada en altamar

El despliegue de barreras mediante barcos convencionales es una práctica habitual cuando se produce un vertido. Sin embargo requiere tripulaciones entrenadas para asegurar un uso eficiente del sistema, ya que es preciso coordinar los esfuerzos de los barcos remolcadores, que deben cooperar en el transporte y despliegue de la barrera. Cuando el vertido adquiere proporciones catastróficas, como en el caso del accidente sufrido en el año 2010 por la plataforma de prospección petrolífera Deepwater en aguas del Golfo de México, los recursos disponibles resultan insuficientes y quedan exhaustos antes de que se pueda dar una respuesta adecuada al accidente [8], [9].

El desarrollo que ha experimentado durante la última década la investigación en automatización de vehículos autónomos y en particular el de los vehículos marinos de superficie [2], conocidos por sus siglas en inglés: USV (Unmmaned Surface Vehicles), hace posible plantear un escenario en que grupos de USVs lleven a cabo el trasporte y despliegue de barreras de modo automático.

Automatizar las maniobras de trasporte y despliegue de barreras permitiría mantener operativos un número más alto de equipos para la recogida del vertido, durante todo el tiempo que fuera necesario, minimizando al mismo tiempo el riesgo para los seres humanos.

Desde el punto de vista del control, el sistema resultante presenta el interés de exigir la coordinación de movimientos entre los USVs que remolcan la barrera. Ésta su vez, actúa como una perturbación que afecta al movimiento de los USVs.

El estudio de escenarios de transporte y despliegue de barreras de control se ha abordado desde dos perspectivas complementarias: De una parte, se están desarrollando modelos a escala para estudiar el comportamiento dinámico del sistema remolcadores-barrera y probar los algoritmos de control desarrollados. Algunos resultados prelimi- nares de estos modelos físicos pueden encontrarse en [6]. De otra se están elaborando modelos matemáticos que permitan orientar los experimentos con los modelos a escala, así como hacer estudios preliminares de los sistemas de control y guiado.

El presente artículo muestra los desarrollos realizados en el contexto de dichos modelos modelos matemáticos, así como algunos de los resultados obtenidos.

Lo que resta del presente artículo se ha estructurado de la siguiente forma: la sección 2 introduce los fundamentos físicos que guían el modelo. La sección 3 describe la estructura de los sistemas de control empleados para gobernar el movimiento de los USVs. La sección 4 muestra algunos resultados obtenidos mediante el modelo propuesto para algunos escenarios simples. Por último, la sección 5 presenta algunas conclusiones y las líneas de trabajo futuras.

\section{Desarrollo de modelos dinámicos}

Se han desarrollado dos modelos matemáticos a partir de primeros principios. El primer modelo describe la dinámica de un USV genérico. Es posible ajustar todos sus parámetros, incluido el modo de propulsión y el de gobierno. El segundo modelo describe la dinámica de una barrera de contención de vertidos. Al igual que en el caso de los USVs, es posible ajustar sus principales parámetros.

Los modelos dinámicos desarrollados han servido para probar y validar sistemas de control que permitan a un equipo de dos o más USVs desplegar y arrastrar una barrera. Un hecho, fácilmente constatable a partir de los modelos, es que es imprescindible el control coordinado de ambos USVs para lograr una dinámica estable. Los sistemas de control se han ido completando a partir de un controlador PID sencillo para la velocidad y un controlador PI para el rumbo.

Los modelos se han probado sistemáticamente en diversos escenarios de arrastre. Los resultados obtenidos hasta ahora, aunque permiten establecer las condiciones fundamentales para el control del sistema, son todavía cualitativos. Se está a la espera de obtener medidas experimentales con barreras de contención reales para ajustar los parámetros de los modelos.

\subsection{Modelo dinámico de un USV}

Se considera el USV propulsado por una fuerza impulsora $\vec{F}_{m}$ producida bien por un motor intraborda, por un motor fuera borda o por un waterjet. En el primer caso, $\vec{F}_{m}$ estará siempre orientada 


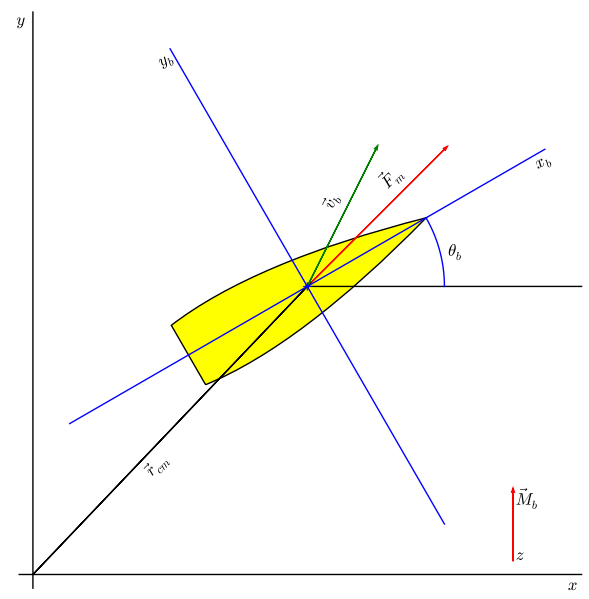

Figura 2: Esquema de un USV con los parámetros empleados para describir su dinámica

en la dirección de la eslora del USV. En los otros dos casos, la dirección de la fuerza impulsora estará acoplada con el gobierno del barco. La fuerza impulsora genera aceleraciones en el barco. Tal y como se han construido los modelos, no existe la posibilidad de dar contra máquina, es decir, no es posible frenar el avance de los USV invirtiendo la fuerza impulsora. La combinación de la fuerza impulsora más el sistema de gobierno (timón) genera un momento M responsable de los giros del USV.

El modelo se completa con las resistencias al movimiento producidas por la fricción. Se han definido coeficientes asociados al desplazamiento en la dirección de la eslora, al desplazamiento en la dirección de la manga y a la rotación del USV. Las fuerzas resistivas se consideran, en primera aproximación proporcionales a la velocidad del barco y a su cuadrado.

Por último, el modelo permite incluir una fuerza externa y su punto de aplicación. Esta fuerza externa se emplea para modelar la tensión ejercida por la barrera de contención sobre el USV durante las operaciones de despliegue o arrastre.

$$
\begin{array}{r}
\left(m_{b}+m_{A \|} \cos \left(\theta_{b}\right)-m_{A \perp} \sin \left(\theta_{b}\right)\right) \cdot a_{b x}= \\
F_{m x}-\mu_{l}\left[v_{b x} \cos \left(\theta_{b}\right)+v_{b y} \sin \left(\theta_{b}\right)\right] \cos \left(\theta_{b}\right) \\
+\mu_{t} l_{s}\left[v_{b y} \cos \left(\theta_{b}\right)-v_{b x} \sin \left(\theta_{b}\right)\right] \sin \left(\theta_{b}\right) \\
-\mu_{l 2}\left[v_{b x} \cos \left(\theta_{b}\right)+v_{b y} \sin \left(\theta_{b}\right)\right]\left|\overrightarrow{v_{b}}\right| \cos \left(\theta_{b}\right) \\
+\mu_{t 2} l_{s}\left[v_{b y} \cos \left(\theta_{b}\right)-v_{b x} \sin \left(\theta_{b}\right)\right]\left|\overrightarrow{v_{b}}\right| \sin \left(\theta_{b}\right)+F_{e x}
\end{array}
$$

Las expresiones generales del modelo desarrollado se muestran en la ecuaciones (1) - (3). Reflejan un modelo sencillo de maniobra en dos dimensiones [5], en el que se han incluido masas añadidas dependientes de la dirección de movimiento del barco $\theta_{b}$. La figura (2) Muestra esquemáticamente un USV con los sistemas de referencia y fuerzas empleados.

$$
\begin{array}{r}
\left(m_{b}+m_{A \| \mid} \sin \left(\theta_{b}\right)+m_{A \perp} \cos \left(\theta_{b}\right)\right) \cdot a_{b y}= \\
F_{m y}-\mu_{l}\left[v_{b x} \cos \left(\theta_{b}\right)+v_{b y} \sin \left(\theta_{b}\right)\right] \sin \left(\theta_{b}\right) \\
-\mu_{t} l_{s}\left[v_{b y} \cos \left(\theta_{b}\right)-v_{b x} \sin \left(\theta_{b}\right)\right] \cos \left(\theta_{b}\right) \\
-\mu_{l 2}\left[v_{b x} \cos \left(\theta_{b}\right)+v_{b y} \sin \left(\theta_{b}\right)\right]\left|\overrightarrow{v_{b}}\right| \sin \left(\theta_{b}\right) \\
-\mu_{t 2} l_{s}\left[v_{b y} \cos \left(\theta_{b}\right)-v_{b x} \sin \left(\theta_{b}\right)\right]\left|\overrightarrow{v_{b}}\right| \cos \left(\theta_{b}\right)+F_{e y} \\
(2) \\
I_{b} \alpha_{b}=M-\mu_{a} l_{s} \omega_{b}+M_{e}
\end{array}
$$

\subsection{Modelo dinámico de las barreras de contención}

Las barreras se han modelado considerándolas como una cadena de elementos rígidos, flotantes, unidos entre sí por articulaciones flexibles. Se describe el movimiento de cada uno de estos elementos sometido a las tensiones ejercidas en sus extremos por los elementos a los que está concatenado. De modo análogo al caso de los barcos, se ha asociado a cada uno de los elementos que conforman la barrera coeficientes de fricción ligados al desplazamiento del elemento en sus direcciones longitudinal y trasversal, y al giro del mismo. Las fuerzas resistivas se consideran, en primera aproximación proporcionales a la velocidad del elemento y su cuadrado. Las ecuaciones siguientes, expresan la dinámica propia de un elemento genérico $(i)$ de la barrera:

$$
\begin{array}{r}
\vec{T}_{i, i+1}-\vec{T}_{i-1, i}-\left(\left|\vec{v}_{i} \cdot \vec{n}_{i}\right| s+\left|\vec{v}_{i} \cdot \vec{p}_{i}\right| q\right) \frac{\vec{v}_{i}}{\left|\vec{v}_{i}\right|} \\
-\left(\left|\vec{v}_{i} \cdot \vec{n}_{i}\right| s_{2}+\left|\vec{v}_{i} \cdot \vec{p}_{i}\right| q_{2}\right) \vec{v}_{i}=m_{A i} \cdot \vec{a}_{i} \\
\left(\vec{T}_{i, i+1} \cdot \vec{n}_{i}+\vec{T}_{i-1, i} \cdot \vec{n}_{i}\right) l-A \omega_{i}=I \alpha_{i} \\
m_{A i}=\left(\begin{array}{cc}
m+m_{a} \cos \left(\theta_{i}\right) & 0 \\
0 & m+m_{a} \sin \left(\theta_{i}\right)
\end{array}\right)
\end{array}
$$

Es preciso añadir una ecuación que refleje la continuidad de la barrera, ligando entre sí elementos contiguos,

$$
\vec{r}_{i}-l \vec{p}_{i}-l \vec{p}_{i+1}-\vec{r}_{i+1}=0
$$

Las ecuaciones (4) - (7) constituyen un conjunto completo de ecuaciones y permiten obtener las 
tensiones en los extremos de todos los elementos de una barrera de longitud arbitraria, si se conocen las fuerzas ejercidas en los extremos de la barrera.

La figura 3 muestra las variables empleadas en el desarrollo de las ecuaciones de la dinámica de la barrera.

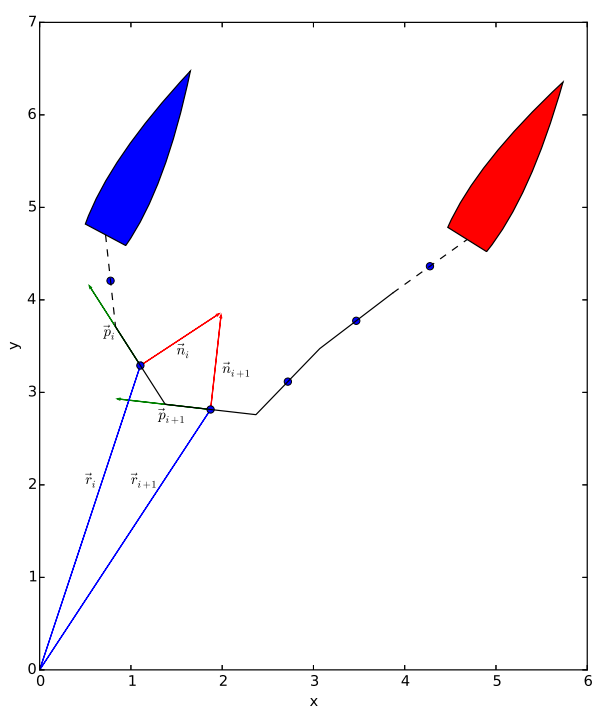

Figura 3: Dos elementos genéricos consecutivos, $(i$ e $i+1$ ), de una barrera mostrando las variables empleadas en las ecuaciones (4) - (7)

Para obtener las fuerzas aplicadas en los extremos de las barreras se han empleado dos aproximaciones distintas.

En la primera de ellas, los elementos situados en los extremos de la barrera se unen directamente a los barcos remolcadores. En este caso, los barcos pasan a constituir un elemento más del sistema, la ecuación (7) se sustituye por una ecuación de cierre entre el último elemento y el barco al que está unido,

$$
\begin{gathered}
\vec{r}_{c m}-\frac{\vec{l}_{s}}{2}-l \vec{p}_{2}-\vec{r}_{2}=0 \\
\vec{r}_{m}-\frac{\vec{l}_{s}}{2}-l \vec{p}_{m}-\vec{r}_{c m}=0
\end{gathered}
$$

Las ecuaciones (8) y (9) se aplican, respectivamente, al barco y al elemento situados en los extremos izquierdo y derecho de la barrera. La fuerza aplicada al extremo de la barrera se calcula como una tensión más.

El la segunda aproximación, más adecuada a la realidad, los extremos de las barreras se unen a los barcos mediante cabos de remolque. En primera aproximación la tensión soportada por el cabo se asocia a la elasticidad de éste,

$$
\begin{array}{r}
T_{c b l}=\frac{1}{\kappa \cdot L} d, d \geq L \\
T_{c b l}=0, d<L
\end{array}
$$

$d$ representa la distancia entre los puntos de anclaje del cabo, L la longitud total del cabo y $\kappa$ es la elasticidad del cable. Dado que el cable no admite esfuerzos cortantes, la orientación del cable da la dirección de $T_{c b l}$, con lo que es posible resolver el sistema barcos - barrera.

\section{Estrategias de control básicas.}

\subsection{Control de rumbo y velocidad de USVs independientes.}

Tanto para el control del rumbo como de la velocidad de un USV aislado, se han implementado controladores PID simples. El control de velocidad, admite alimentación feedforward. Esto facilita el acercamiento de la velocidad a sus valores de consigna, disminuyendo el esfuerzo de control y atenuando el efecto del acoplamiento entre propulsión y rumbo. La figura 4 muestra los esquemas del sistema de control de un USV aislado. Es importante resaltar que aunque los sistemas de control implementan un PID completo, no siempre se usan todos los términos. Así por ejemplo, el control de rumbo no emplea acción integral.

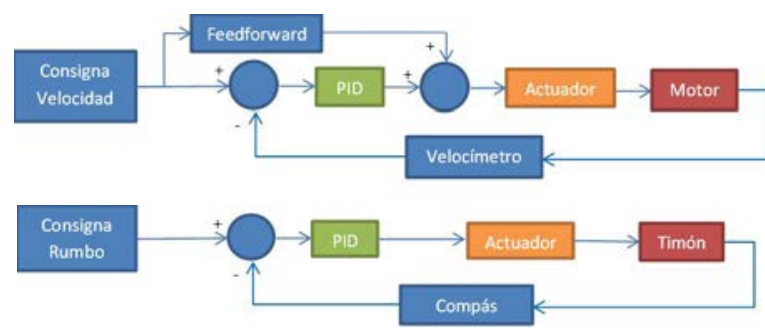

Figura 4: Esquema de los sistemas de control de velocidad y rumbo para un USV

\subsection{Control de dos USVs arrastrando una barrera de contención.}

No es posible conseguir una maniobra estable, en un escenario de arrastre, a menos que se empleen algunas estrategias básicas de control cooperativo. Una posible aproximación es modificar los controladores de los USVs de modo que adapten los valores de rumbo y posición a la situación del escenario de arrastre. En el caso presente, se ha construido una arquitectura de control que emplea un juego de PIDs paralelos para cada variable de control. 
Para el control de velocidad, el valor introducido al actuador combina la contribución de dos errores:

En primer lugar el error de velocidad, entendiendo por tal la diferencia entre la velocidad actual del USV y la velocidad consignada para el desplazamiento del sistema completo formado por los dos USVs y la barrera que remolcan.

En segundo lugar, se ha introducido un error al que se ha denominado error de alineación. Se considera que los barcos navegan correctamente alineados cuando sus rumbos son iguales y perpendiculares a la línea que une sus centros de masas. En cualquier otra situación se considera que los barcos navegan con un error de alineación. A efectos de control, ese error se estima calculando un rumbo medio instantáneo a partir del rumbo de los dos barcos y un vector unitario en dicha dirección,

$$
\begin{aligned}
\theta_{m} & =\frac{\theta_{i}+\theta_{d}}{2} \\
\vec{u}_{m} & =\left(\cos \theta_{m}, \sin \theta_{m}\right)
\end{aligned}
$$

Adicionalmente, Se calcula un vector unitario $\vec{u}_{c m}$ en la dirección que une el centro de masas del USV con el de su compañero. El error de alineamiento se calcula como el producto escalar de ambos vectores,

$$
e_{a l}=\vec{u}_{m} \cdot \vec{u}_{c m}
$$

Para el control de rumbo se considera la contribución de tres errores distintos: En primer lugar el error de rumbo entendiendo por tal la diferencia entre el rumbo actual del USV y el rumbo consignado para el desplazamiento del sistema completo formado por los dos USVs y la barrera que remolcan. En segundo lugar el error de alineamiento descrito más arriba. En el caso del control de rumbo, la corrección es de distinto signo según el USV esté situado en un extremo u otro de la barrera que remolca. Para tener este efecto en cuenta se ha añade al error de alineamiento el signo del producto vectorial: $\vec{u}_{c m} \times \vec{u}_{m}$.

Por último, se establece una distancia de consigna que deben mantener los USV entre sí cuando navegan en paralelo arrastrando la barrera. El error empleado, al que se ha denominado error de distancia de navegación, establece la diferencia entre la distancia actual de los centros de masa de los dos USVs y la distancia de consigna. Aunque esta distancia se establece sin tener en cuenta la orientación relativa de los USVs, la combinación con el control de alineamiento hace que tienda a convertirse en una distancia lateral con los USVs navegando en paralelo. La figura 5 muestra posiciones sucesivas de una barrera arrastrada por dos USVs.
Los barcos parten del reposo con rumbo norte y una separación entre ellos de 23 metros. su objetivo es navegar a una velocidad de dos nudos en dirección este, separados entre sí cinco metros. Las líneas en ' $\mathrm{T}$ ' muestran la distancia que une sus centros de masa y su perpendicular. Es posible observar cómo los barcos van alinéandose a la vez que ajustan la distancia entre ellos y se acercan al rumbo consignado. Lógicamente esto exige que durante la maniobra de giro y aproximación la velocidad del barco situado a la izquierda sea mayor que la del situado a la derecha.

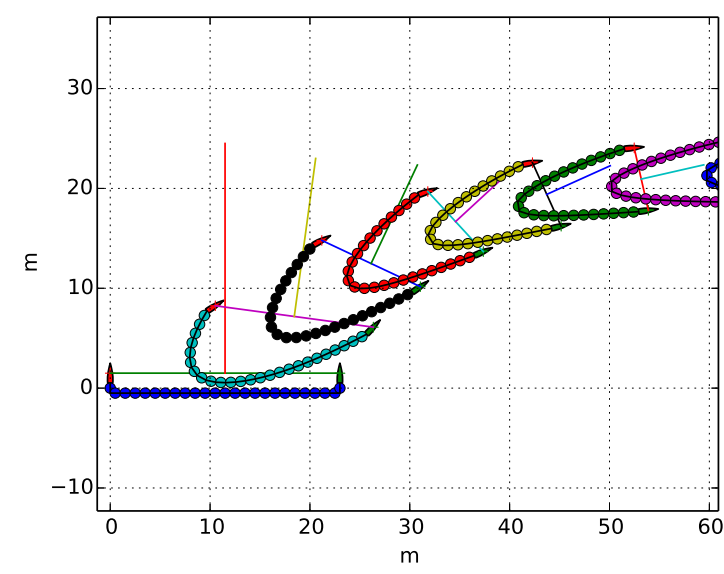

Figura 5: Detalle de la maniobra de alineamiento entre dos USVs mientras realizan un viraje de $90^{\circ}$. La cadena de círculos representa la barrera, los triángulos en los extremos la posición y orientación de los USVs

Los errores descritos contribuyen al esfuerzo de control a través de sus propias constantes, proporcional, integral y derivativa. El ajuste de dichas constantes se ha realizado empíricamente en los ensayos preliminares con el modelo. Es evidente que los errores van a competir entre sí y que se llegará a una situación de equilibrio en la que los valores de offset son inevitables. Por ejemplo, si se desea remolcar la barrera en línea recta siguiendo un rumbo determinado los USVs se desplazarán en el equilibrio con sus proas apuntando en una dirección próxima al rumbo fijado sin alcanzarlo, el valor de equilibrio dependerá de la distancia de consigna entre los barcos y la tensión que la barrera ejerce sobre ellos.

El cálculo de los errores de alineación y distancia entre USVs, exige que se emplee información de ambos vehículos. El control está distribuido; cada USV coopera con la corrección ejercida por su propio sistema de control a la realización de la maniobra.

Los USVs comparten información a través de un 
enlace de radio. Los datos compartidos son la posición, el rumbo y las velocidades lineal y angular instantáneas. Para el cálculo de los errores, es suficiente con conocer la posición y el rumbo del otro USV. Las velocidades son compartidas para que cada USV pueda implementar un modelo elemental del movimiento de su compañero. Así, los errores de alineación y distancia pueden ser estimados entre una recepción y la siguiente. La figura 6 muestra el esquema de los controladores empleados por los USVs para tareas de cooperación en el arrastre de barreras.

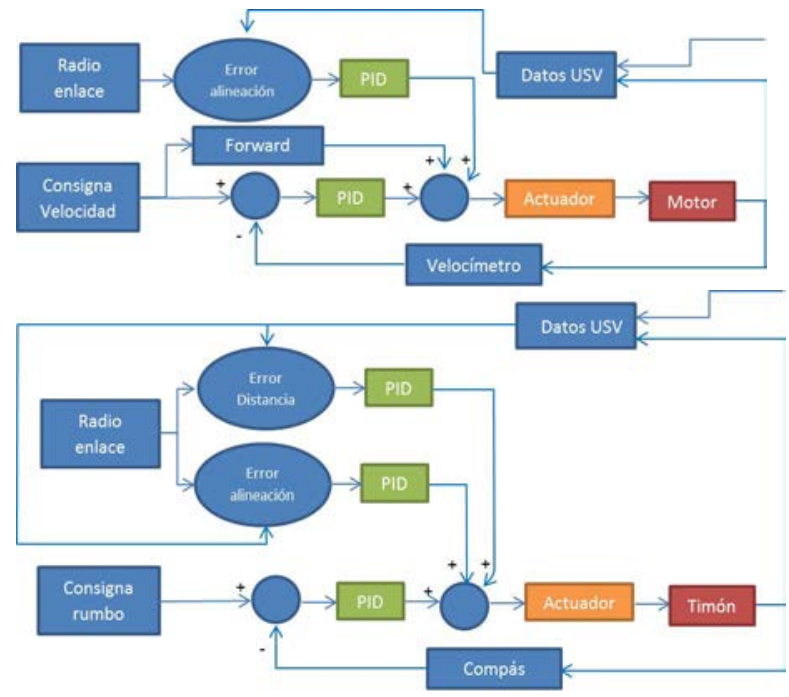

Figura 6: Esquema de los sistemas de control de velocidad y rumbo para dos USVs cooperando en el arrastre de una barrera

\section{Resultados para algunos escenarios simples.}

Los escenarios descritos en esta sección se han realizado empleando en todos los casos las mismas condiciones para los barcos y las barreras. La tabla 1 resume los valores utilizados para los principales parámetros del modelo.

Es interesante notar en primer lugar que dichos valores son arbitrarios y, aunque aproximados, no corresponden con medidas reales. Por otro lado no se están empleando términos cuadráticos a la resistencia al movimiento ni tampoco masas añadidas.

El primer escenario probado consiste en el remolque en línea recta de una barrera de $25 \mathrm{~m}$, llevada a cabo por dos USVs. El sistema parte del reposo, la barrera está completamente desplegada en sentido este-oeste y los barcos deben remolcarla en dirección norte con una separación final entre los extremos de 5 metros. La velocidad de consigna para los USVs es de 2 nudos. La figura 7 muestra la evolución de la posición y configuración de la

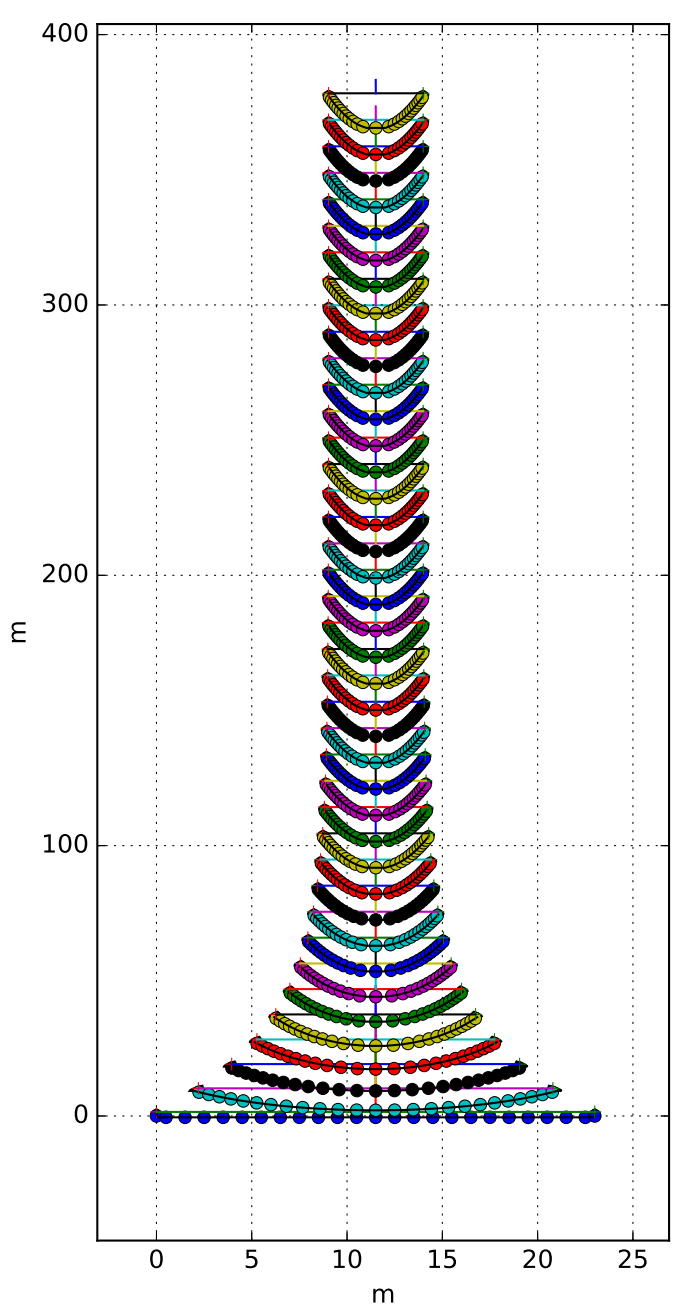

Figura 7: Ejemplo de maniobra de aproximación y remolque en línea recta entre dos USVs. Distancia inicial entre los barcos: $25 \mathrm{~m}$. Consigna para la distancia de navegación: 5 metros. Las posiciones mostradas corresponden a intervalos de tiempo de simulación de $10 \mathrm{~s}$.

barrera en intervalos sucesivos de tiempo de $10 \mathrm{~s}$. El sistema emplea unos cien metros desde la configuración inicial hasta que empiezan a navegar a la distancia de consigna. El movimiento de los barco es simétrico y el control de alineamiento entre ellos no actúa ya que navegan alineados desde el principio.

El siguiente escenario representa un maniobra combinada de giro y acercamiento. Las condiciones iniciales son las mismas del escenario anterior pero ahora la consigna de rumbo es 045 (NE). En este escenario se combinan todos los esfuerzos de control descritos en la sección 3. La figura 8 muestra la evolución de la posición y configuración de la barrera. Este escenario muestra algunos elementos interesantes del sistema: la combinación de las acciones de control de rumbo, alineamiento y dis- 


\begin{tabular}{|c|l|l|}
\hline \multicolumn{2}{|c|}{ Cuadro 1: Parámetros del modelo } \\
\hline \multicolumn{1}{|c|}{ USV } & Elemento Barrera \\
masa $(\mathrm{Kg})$ & 500 & 2 \\
momento de inercia $(\mathrm{Kgm})$ & 1000 & 0.02 \\
eslora $(\mathrm{m})$ & 5 & 1 \\
manga $(\mathrm{m})$ & 2 & 0.1 \\
coef. resitencia despl longitudinal $(N s / m)$ & 1000 & - \\
coef resistencia desplaz. lateral $\left(N(s / m)^{2}\right)$ & 10000 & 50 \\
coef. resitencia despl longitudinal $\left(N(s / m)^{2}\right)$ & 0 & 0 \\
coef resistencia desplaz. lateral $(\mathrm{Ns} / \mathrm{m})$ & 0 & - \\
Potencia max $(\mathrm{CV})$ & 50 & - \\
Tipo de Gobierno & waterjet & - \\
\hline
\end{tabular}

tancia de navegación entre los USVs, hace que el barco situado a la izquierda de la barrera adquiera una mayor velocidad durante la fase transitoria del movimiento. Esto tiene como consecuencia que se alcance la situación estacionaria en una distancia significativamente menor que en el escenario anterior. El barco de la derecha por el contrario retarda su avance, acelerando más despacio, a la espera de su compañero.

El escenario pone de manifiesto el carácter cooperativo del esfuerzo de control, basado en el intercambio de información de estado llevado a cabo entre los dos barcos. Es destacable que se trata de un sistema puramente reactivo: no hay ninguna estructura por encima de los controladores de los USVs encargada de coordinarlos.

La figura 9 muestra en detalle los últimos estadios de la simulación del escenario descrito. Es fácil apreciar cómo los barcos navegan en paralelo, perpendiculares a la línea que une sus centros de masas y con el rumbo de consigna prefijado. Han alcanzado sus consignas de rumbo, velocidad, alineamiento y distancia de navegación y permanecerán en estado estacionario mientras no se alteren dichas consignas.

\section{Conclusiones y trabajos futuros}

El presente trabajo presenta algunos resultados preliminares alcanzados con los modelos desarrollados para el estudio de la dinámica y el control del despliegue de barreras de contención de vertidos. El objetivo final es desarrollar una herramienta que permita anticipar resultados al despliegue de sistemas con USVs y barreras reales.

Los modelos desarrollados han permitido probar en simulación esquemas simples de control cooperativo para la coordinación del movimientos de dos USVs, que participan en el transporte y despliegue de una barrera de contención de vertidos marinos.

Se han realizado pruebas, empleando distintos es- cenarios, condiciones de navegación y parámetros de la barrera y los USVs. En este artículo se presentan solo algunos casos básicos que permiten ilustrar el funcionamiento del sistema y la efectividad de las estrategias de control aplicadas.

Los resultados muestran que es posible con unos esquemas simples de control y el intercambio de información entre los USVs conseguir que el sistema alcance un estado estacionario con el rumbo y velocidad prescrito por los valores de consigna.

Entre los posibles trabajos futuros previstos cabe destacar:

- La consideración de perturbaciones debidas a corrientes y mareas. Su inclusión es sencilla Usando para los términos resistivos de los distintos elementos del sistema la velocidad relativa respecto al agua [5]. Las corrientes o las mareas se modelan como un campo de velocidades asociado al medio. Los resultado de algunos ensayos preliminares muestran la necesidad de planificar cuidadosamente la trayectoria del sistema cuando hay corrientes presentes.

- La realización de experimentos con los modelos a escala de USVs y barreras de contención reales. Tanto para el ajuste de parámetros del modelo como para la comprobación experimental del funcionamiento de los algoritmos de control propuestos. A tal efecto, se está completando la puesta a punto de un sistema experimental.

- El desarrollo de un sistema de planificación de trayectorias que permita optimizar la maniobra de despliegue y confinamiento de un vertido. Se han desarrollado también algunos ensayos preliminares empleando curvas de Bézier para estudiar trayectorias que permitan el aislamiento mediante barreras de un buque atracado. Los resultados obtenidos son prometedores. 


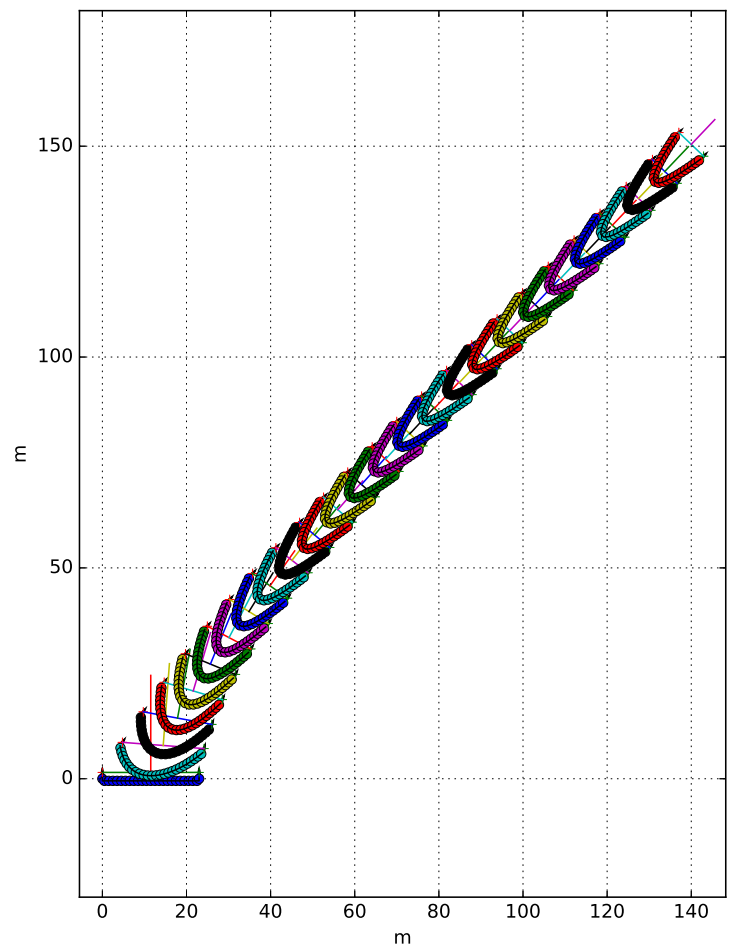

Figura 8: Ejemplo de maniobra de aproximación y remolque entre dos USVs. Distancia inicial entre los barcos: $25 \mathrm{~m}$. Consigna distancia de navegación: 5 metros. Consigna de rumbo: 045 (NE). Las posiciones mostradas corresponden a intervalos de tiempo de simulación de $10 \mathrm{~s}$.

Una línea futura interesante, sería el desarrollo de un sistema de coordinación que permitiera desplegar flotas completas de USVs remolcando barreras de contención para su uso en vertidos de gran envergadura.

\section{Agradecimientos}

Los autores agradecen la financiación recibida de los proyectos: DPI2013-46665-C02-1-R y DPI2013-46665-C02-2-R: "Sistema Autónomo para la Localización y Actuación ante Contaminantes en el Mar", de la que es parte el presente trabajo.

\section{Referencias}

[1] Ray R Ayers, Robert W Patterson, and Wayne F Simpson. Containment boom system, February 3 1987. US Patent 4,640,645.

[2] Jesús M. de la Cruz García, Joaquín Aranda Almansa, and José M. Girón Sierra. Automática marina: una revisión desde el punto

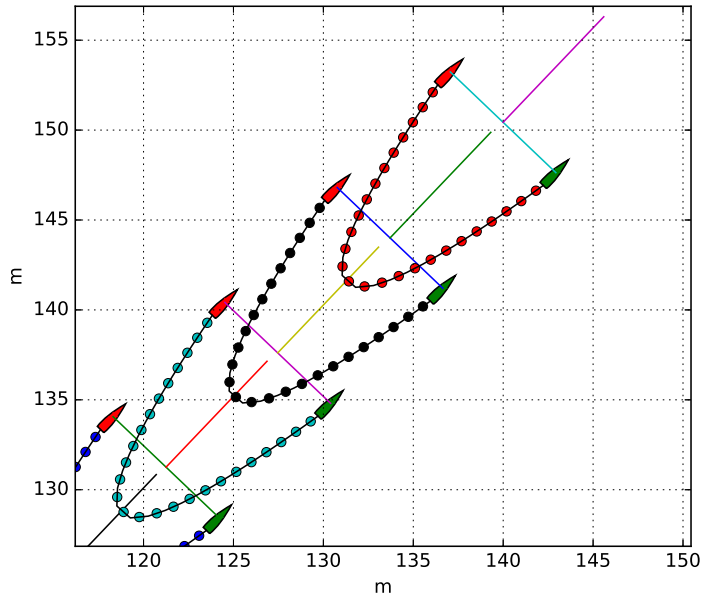

Figura 9: Detalle del final de la maniobra representada en la figura 8

de vista del control. Revista Iberoamericana de Automática e Informática Industrial RIAI, 9(3):205 - 218, 2012.

[3] European Maritime Safety Agency. Action Plan for Response to Marine Pollution from Oil and Gas Installations, 2013.

[4] Merv Fingas. The basics of oil spill cleanup. CRC Press, 2012.

[5] Thor I Fossen. Marine control systems: guidance, navigation and control of ships, rigs and underwater vehicles. Marine Cybernetics Trondheim, 2002.

[6] Jose M. Giron-Sierra, Alina T. Gheorghita, Guillermo Angulo, and Juan F. Jimenez. Preparing the automatic spill recovery by two unmanned boats towing a boom: Development with scale experiments. Ocean Engineering, 95:23 - 33, 2015.

[7] William F Gunderson III. Floating containment boom, April 7 1992. US Patent $5,102,261$.

[8] Wassel R. Lessons from the macondo well blowout in the gulf of mexico. The Bridge, 42(3):46-52, Fall 2012.

[9] Mark Reed, Narve Ekrol, Henrik Rye, and Liam Turner. Oil spill contingency and response (oscar) analysis in support of environmental impact assessment offshore namibia. Spill science 85 technology bulletin, 5(1):29-38, 1999. 